\title{
Schmidt type optical system for the KLYPVE-EUSO UHECR detector
}

\section{P. Sandri}

OHB-Italia $\operatorname{SpA}$

via Arno 108, 50019 Osmannoro, Sesto Fiorentino (FI) - Italy

E-mail: psandri@cgspace. it

\section{S. Sharakin ${ }^{1}$}

Skobeltsyn Institute of Nuclear Physics, Lomonosov Moscow State University

1(2), Leninskie gory, GSP-1, Moscow 119991, Russian Federation

E-mail: sharakinemail.ru

\section{P. Mazzinghi}

Istituto Nazionale di Ottica

Largo Enrico Fermi, 6 - 50125 Firenze (Italy)

E-mail: piero.mazzinghilino.it

\section{Y. Takizawa}

RIKEN

2-1 Hirosawa, Wako, Saitama 351-0198, Japan

E-mail: takkyeriken.jp

for the JEM-EUSO Collaboration

\begin{abstract}
We present the optical design of the baseline solution for the telescope of the KLYPVE-EUSO UHECR experiment. The adopted optical layout is a Schmidt camera covering a field of view of $40^{\circ}$ with an entrance pupil diameter of $2.5 \mathrm{~m}$, a 4 m diameter spherical mirror and a focal length of 1.7 $\mathrm{m}$. The resulting very fast relative aperture (F/\# 0.7$)$ minimizes the detector size and its obstruction, resulting in a throughput of $70 \%$ over the entire field of view. The optical design, with an optimized shape of the corrector plate, allows for a correction of the geometric and the chromatic aberrations in the spectral range from $337 \mathrm{~nm}$ to $391 \mathrm{~nm}$. The resulting nominal polychromatic RMS spot size is well inside the dimension of pixel size $(3 \mathrm{~mm})$ of the multi-anode PMT all over the field of view, providing a ground resolution of about $750 \mathrm{~m}$ from the ISS orbit. The maximum geometric distortion is below $5 \%$. The axial length of the camera and the position of the aperture stop have been optimized in order to meet the stringent requirements on vignetting and throughout.
\end{abstract}

\section{${ }^{1}$ Speaker}




\section{Introduction}

It is known from literature, Ref. [1]. to [4]., that the Schmidt telescope harmonizes two conflicting requirements in optical systems: a wide as possible field of view (FoV) and a fast as possible relative aperture F/\#. Moreover its simple layout consisting of a few number of optical elements make the Schmidt camera the preferred solution for wide FoV imaging telescopes (Ref. [5]., [6]., [7].). The limited possibility in the correction of the spherochromatism is generally compensated by the narrow band operating spectral bandwidth. The classic layout of the Schmidt telescope consists of a spherical mirror, of a corrector plate and of a focal surface. The aperture stop, placed at the centre of curvature of the spherical mirror, provides uniform images over a wide spherical focal surface concentric with the mirror. As each image suffers of a large amount of spherical aberration, Schmidt (Ref. [8].) introduced into the aperture stop a thin, nearly plane-parallel plate, called corrector plate. The aim of this plate is to pre-correct the plane wave-fronts entering the system thus compensating the spherical aberration introduced by the spherical mirror. A remarkable advantage of the Schmidt telescope consists in having the image surface not facing the object plane avoiding the radiation from out-of-field sources (e.g. scattered out-of-field Earthshine) to directly impinge on the image surface. This feature, combined with the low number of optical elements, results in a particularly efficient suppression of the stray light both in-field and out-of-field.

The paper reports the optical design of a compact Schmidt telescope covering a FoV of $40^{\circ}$ with an entrance pupil diameter of $2.5 \mathrm{~m}$, a $4 \mathrm{~m}$ diameter mirror and a focal length of $1.7 \mathrm{~m}$ optimized both for image quality and throughput.

\section{Requirements}

The fluorescence detector of ultra-high energy cosmic rays (UHECRs, energies $\geq 50 \mathrm{EeV}$ ) of the KLYPVE-EUSO project will be installed on the Russian Segment of the International Space Station and will operate at an orbit $\mathrm{H} \approx 450 \mathrm{~km}$ from the Earth's surface. The requirement for each detector pixel, with size $\mathrm{p}=3 \mathrm{~mm}$, of the multi anode photo multipliers is to cover an atmosphere portion corresponding to a ground sampling distance ( $G S D$ ) on Earth between $0.5 \mathrm{~km}$ and $1 \mathrm{~km}$. The required entrance pupil diameter (ENPD) is $2.5 \mathrm{~m}$ and the focal length $f$ results:

$$
f=\frac{p \cdot H}{G S D}=1750 \mathrm{~mm}
$$

for $\mathrm{GSD}=770 \mathrm{~m}$. The relative aperture is very fast, $\mathrm{F} / \# 0.7$, and the covered FoV is $40^{\circ}$ with an operating spectral range extending from $337 \mathrm{~nm}$ to $391 \mathrm{~nm}$ (see Table 1).

\begin{tabular}{|c|c|}
\hline Parameter & Value \\
\hline FoV & $40^{\circ}$ \\
\hline Entrance pupil diameter & $2.5 \mathrm{~m}$ \\
\hline Effective focal length $f$ & $1750 \mathrm{~mm}$ \\
\hline Spectral range & $337-391 \mathrm{~nm}$ \\
\hline Fraction of unvignetted rays & Maximum value $\geq 0.75$; Minimum value $\geq 0.67$ with $\gamma_{\text {vig }} \geq 14^{\circ}(*)$ \\
\hline Diameter of corrector & $2.5 \mathrm{~m}<\mathrm{D}<2.6 \mathrm{~m}$ \\
\hline Shape of the mirror & $\begin{array}{l}\text { - Baseline: spherical mirror } \\
\text { - Advanced: spherical mirror in central part with aspheric petals }\end{array}$ \\
\hline Diameter of the mirror & $4 \mathrm{~m}$ \\
\hline Polychromatic RMS spot & $<3 \mathrm{~mm}$ for all the $\mathrm{FoV}$ \\
\hline Ground sampling distance & $0.5 \mathrm{~km}<G S D<1 \mathrm{~km}$ \\
\hline
\end{tabular}

Table 1. Requirements of the Schmidt camera for the KLYPVE-EUSO. 
Note $(*): \quad \gamma_{v i g} \quad$ is the FoV semi-angle where the decreasing effect starts.

\section{Optical design and performances of the baseline solution}

We started from a classic layout designing with Zemax OpticStudio (Ref. [9].) a spherical mirror, a corrector plate and a spherical focal surface concentric with the mirror, placing the aperture stop on the frontal surface of the corrector plate. We used a sequential merit function for RMS spot radius performing an optimization by using as variables the shape of the corrector with both surfaces set as even aspheres, the radius of curvature of the mirror, the distance between the corrector and the mirror and the back focal length. The radius of curvature of the focal surface is linked to the radius of curvature of the mirror with a pick-up solve. The thickness of the corrector plate is used as a variable in the final phases of the optimization.

As the required average angular resolution is $3.8 \operatorname{arcmin}=\arctan (\mathrm{GSD} / \mathrm{H})$ we are far from the diffraction limit and the geometric optics approximation results appropriate. Authors of Ref. [10]. identified a potential lack of precision of commercial software in the ray-tracing due to the use of approximated polynomial function in the definition of the aspheric corrector. As a consequence of this imprecision, according to Ref. [10]., the axial spherical aberration might be not perfectly corrected after optimization. This is not a limiting issue in the design of the large Schmidt telescope for our application as the resulting error due to this imprecision results inside the required angular resolution. Besides it is necessary to take into account, as stated in Ref. [4]., that the complete correction of the spherical aberration can be achieved for monochromatic light and for one angular field position while in wide angle telescopes it is necessary to reduce the secondary monochromatic aberrations occurring at large field angles at the expenses of the image quality on axial FoV. The material for the corrector plate is PMMA-000 a special Grade UV-transmitting poly methyl methacrylate (by Mitsubishi Rayon Co. LTD, Japan) presenting an internal transmission higher than $90 \%$ for a thickness of $15 \mathrm{~mm}$ and a refractive index of approximately 1.5 in the considered spectral range. Plots of the refractive index and of the internal transmission as a function of the wavelength are available in Ref. [11]. and [12].. The central obstruction generated by the focal surface (FS) is a limiting element in the throughput of the design. The smaller the focal length the less the obstruction. Being the FoV and the focal length fixed requirements for the optical design the throughput for the axial field of view results:

$$
\text { througput }=1-\frac{\text { area of the focal surface }}{\text { area of the entrance pupil }}=1-\frac{d_{F S}^{2}}{E N P D^{2}}=0.74
$$

where the diameter $d_{F S}$ of the focal surface is given by:

$$
d_{F S}=2 \cdot f \cdot \tan \left(\frac{F O V}{2}\right)=1273 \mathrm{~mm}
$$

While the obstruction for the axial FoV is determined only by the first order properties of the telescope, the throughput in-field strongly depends on the vignetting function, mainly because the diameter of the mirror and of the corrector plate are fixed to be respectively $4 \mathrm{~m}$ and less than $2.6 \mathrm{~m}$ for manufacturing reasons. In order to optimize the overall throughput of the telescope we reduced the vignetting at the edge of the field by compacting the axial length of the telescope and optimizing the position of the aperture stop allowing it to span in axial position from in front to behind the corrector plate. The corrector plate introduces chromatic aberrations, mainly spherochromatism. The choice of the optimal form of the plate is determined by the best balance for spherochromatism achieved in the sequential ray-tracing and 
optimization phase and checked with the analysis of the spot diagrams and aberrations plots. Many authors have deeply investigated the design and the optimization of the corrector plate for a Schmidt telescope, e.g. Ref. [3]., [10]., [13].. In our design we used high order terms of the even asphere from $2^{\text {nd }}$ to $16^{\text {th }}$, including in the merit function a constraint on the mass and on the shape of the corrector plate to limit the bending at the edges.

Fig. 1 shows the optimized baseline solution. The different colours of the ray-tracing correspond to different angles of the FoV. The position of the aperture stop, behind the corrector plate, has been identified during the optimization by harmonizing the need of reducing the vignetting at the edge of the field and the necessity of minimizing the aberrations. As the spherical mirror leaves uncorrected transversal aberrations in-field, a great part of the correction is addressed to the corrector plate which therefore presents a consistent a bending at the edge $(\approx 15 \mathrm{~cm})$. The diameter of the corrector plate is $2.6 \mathrm{~m}$ with a mass of $76.6 \mathrm{~kg}$. The corrector plate presents a central hole with a diameter of $10 \mathrm{~cm}$ simplifying the manufacturing process. In fact the plate can be conceived as composed by many identical radial slices (e.g. 12) moulded on a cast and then assembled together with a mechanical holder or through cementing on the lateral sides. The central hole might also represent the access for other instruments, e.g. a Lidar. The diameter of the central hole in the corrector plate is dimensioned such that not to impact on the RMS spot size, as shown in Fig. 2. The maximum geometric distortion results less than 5\% allowing to have a diameter of the focal surface of $1.27 \mathrm{~m}$ in accordance with the above formula (3).

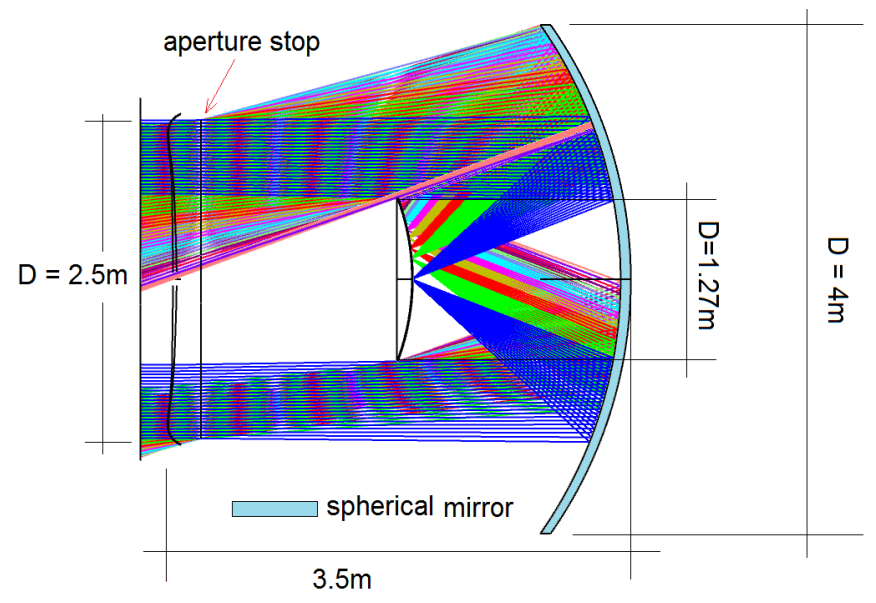

Fig. 1. Sectional view of the baseline layout of the Schmidt camera.

Fig. 2 reports the polychromatic RMS spot radius for the rays refracted by the corrector (solid curve) and for the rays passing through the central hole of the corrector (dotted curve). The right vertical axis of Fig. 2 gives the corresponding half ground resolution (1/2 GSD) in meters.

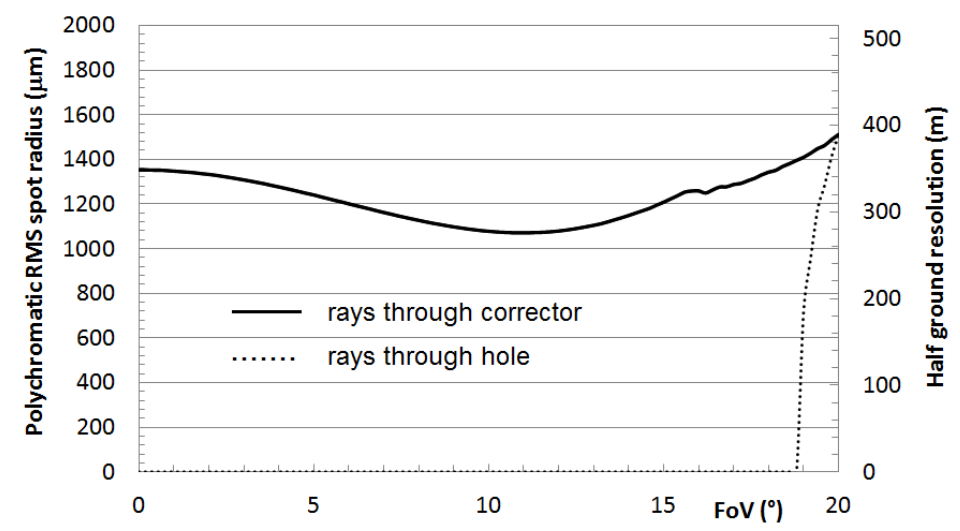


Fig. 2. Spot radius and corresponding half ground resolution as a function of the field angle for the baseline solution.

To check potential lack of accuracies in the ray tracing we compared the polychromatic spot diagrams calculated with the sequential and the non-sequential modalities of Zemax OpticStudio obtaining a good agreement in shape and dimensions (Fig. 3). The non-sequential model which includes the contributor of the surface micro-roughness of the optical items (modelled with the analytical ABg model, see Ref. [14]., [15]., [16]., [17]., [18].) and of the mechanical structure (modelled with Lambertian scattering) allowed us to evaluate the straylight level. The calculated ratio of the in-field stray-light irradiance due to surface microroughness $B_{\text {stray }} \quad$ (photons $\mathrm{cm}^{-2} \mathrm{~s}^{-1}$ ) on the focal plane to the incident mean irradiance $B_{\text {inc }}$ results $\frac{B_{\text {stray }}}{B_{\text {inc }}} \approx 10^{-5}$.

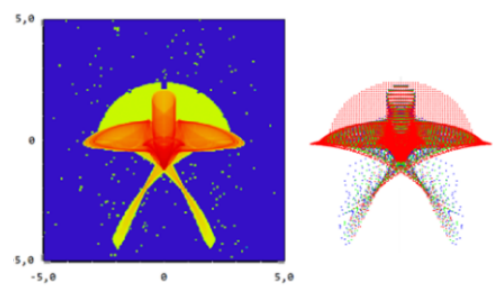

Fig. 3. Polychromatic spot diagrams for $\mathrm{FoV}=20^{\circ}$ traced with the non-sequential/sequential (left/right) modalities of Zemax OpticStudio.

\section{A hint to an advanced solution}

The large dimension of the mirror requires that it is deployable in order to fit the limitation of the launcher fairing diameter and for this reason the mirror is conceived to be segmented. This allows to optimize the shape of mirror (e.g. Ref. [19]., [20]., [21].) in order to achieve a better correction of the aberrations and an easier shape of the corrector from a manufacturing and an integration point of view. When conceiving the mirror as a spherical shape for a diameter up to $3 \mathrm{~m}$ provided with an outer corona with slightly aspheric petals, the profile of the corrector results much less bended at the edges. Left panel of Fig. 4 shows the layout of the advanced solution. The aperture stop is placed in front of the corrector plate. Right panel of Fig. 4 compares the sectional views of the corrector plate for the baseline and for the advanced solution. The diameter of the corrector plate is $2.6 \mathrm{~m}$ with a mass of $66.4 \mathrm{~kg}$ for the advanced solution, $10 \mathrm{~kg}$ less with respect to the baseline one. Due to the more effective correction of the transversal aberrations provided by the aspheric outer corona, the central hole in the corrector can be enlarged to $18 \mathrm{~cm}$, without impacting on the polychromatic RMS spot size (Fig. 5). The larger the central hole, the easier the manufacturing process of the corrector. 

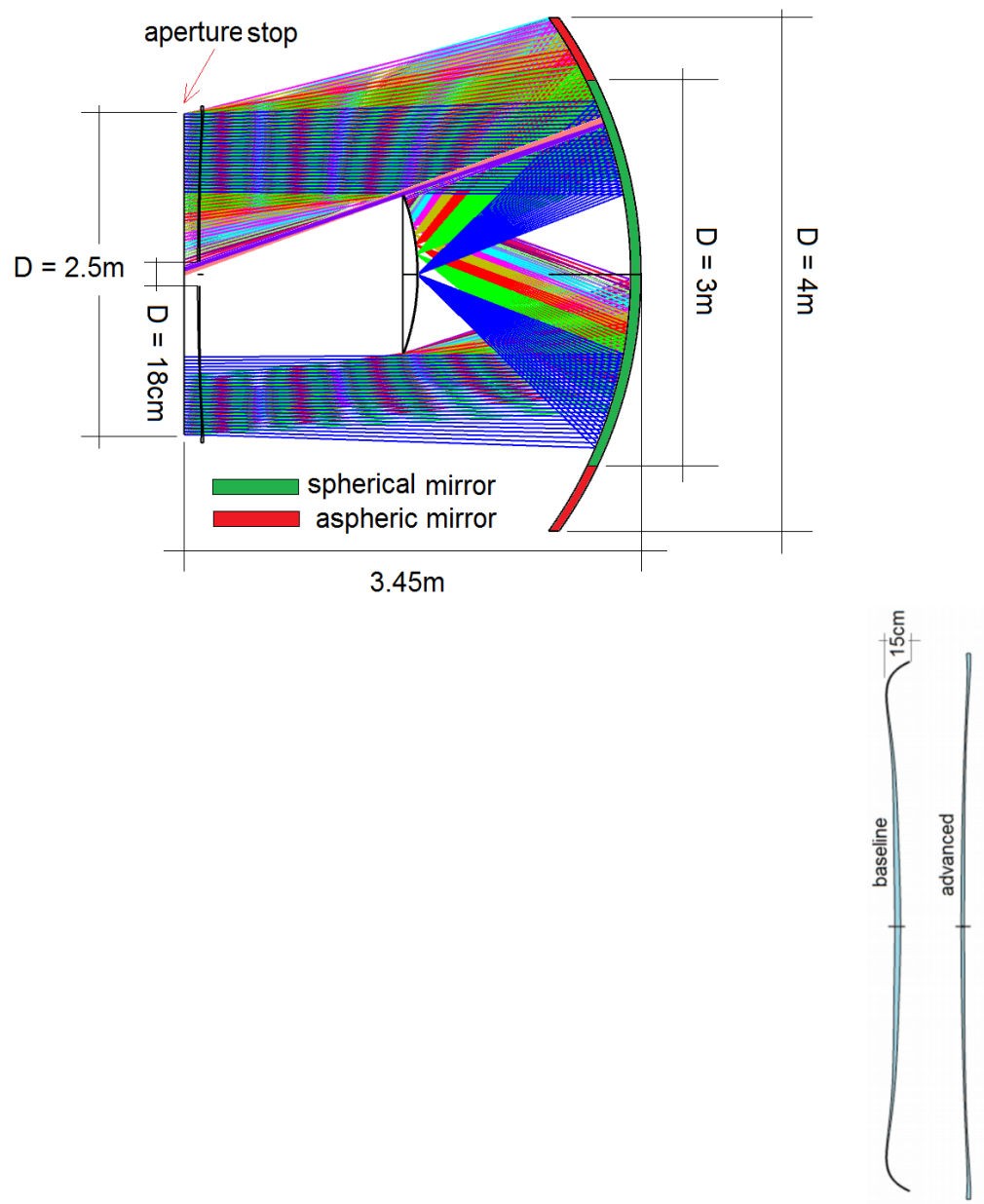

Fig. 4. Left: Sectional view of the advanced layout of the Schmidt camera. Right: Sectional view of the corrector plate for the baseline/advanced (top/bottom) solutions.

Fig. 5 shows the polychromatic RMS spot radius and the corresponding half ground resolution as a function of the FoV. The baseline and advanced solutions are compared in terms of vignetting and geometric distortion in Fig. 6 and in terms of polychromatic ensquared energy collected on an area of $3 \times 3 \mathrm{~mm}^{2}$ in Table 2 .

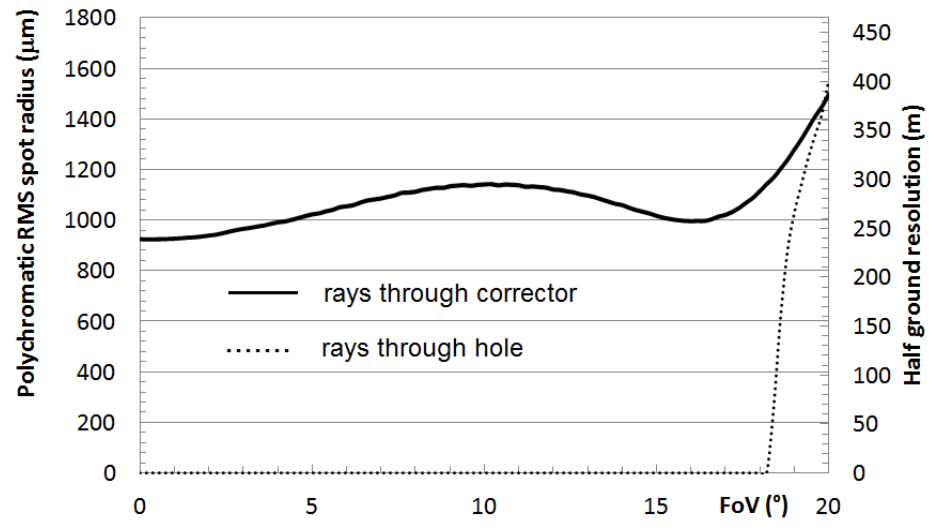

Fig. 5. Spot radius and corresponding half ground resolution as a function of the field angle for the advanced solution. 

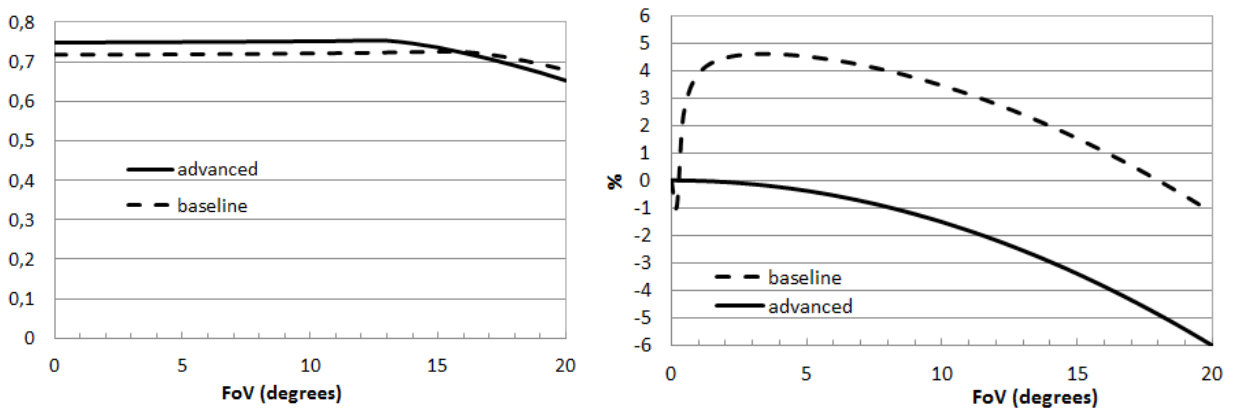

Fig. 6. Left/Right: fraction of unvignetted rays/geometric distortion of baseline (dashed curve) and advanced (solid curve) solution.

\begin{tabular}{|r|r|r|}
\hline $\begin{array}{r}\text { FoV } \\
(\text { degrees })\end{array}$ & $\begin{array}{r}\text { EE - Baseline } \\
\text { solution }\end{array}$ & $\begin{array}{r}\text { EE-Advanced } \\
\text { solution }\end{array}$ \\
\hline 0 & 0.7293 & 0.9153 \\
\hline 5 & 0.8061 & 0.8756 \\
\hline 10 & 0.8965 & 0.8842 \\
\hline 15 & 0.8747 & 0.9321 \\
\hline 20 & 0.7089 & 0.7914 \\
\hline
\end{tabular}

Table 2. Polychromatic ensquared energy (EE) on a $3 \times 3 \mathrm{~mm}^{2}$ for the baseline and the advanced solutions.

\section{Discussion and Conclusions}

The presented telescope results feasible thanks to the progress reached in the construction of deployable and active mirrors. Two ESA projects have recently explored the possibility to develop large lightweight and deployable space mirrors. The first ALC (Advanced Lidar Concept) studied the general concept of a deployable, lightweight, $4 \mathrm{~m}$ aperture space born telescope for Lidar applications (Ref. [19]., [20]., [21].). The second LATT (Large Aperture Telescope Technology) extended the study producing a breadboard of a $400 \mathrm{~mm}$ active mirror, 1 $\mathrm{mm}$ thick, in Schott Zerodur (Ref. [22].) coupled to a carbon fibre reinforced plastic backplane by 19 actuators controlling the optical surface (Ref. [23].).

The presented baseline and advanced solutions of the telescope for the KLYPVE-EUSO instrument allow for a remarkable exposure in the detection of ultra-high energy cosmic rays covering both hemisphere thanks to the orbit of the ISS. Fig. 7 compares the exposure of the two presently operating observatories, the TA (Ref[24].) in Utah and the Auger (Ref. [5].) in Argentina, with the expected exposure of EUSO based on Fresnel lenses Ref. [12]. and on a Schmidt camera, and with the exposure of JEM-EUSO (Ref. [25].).

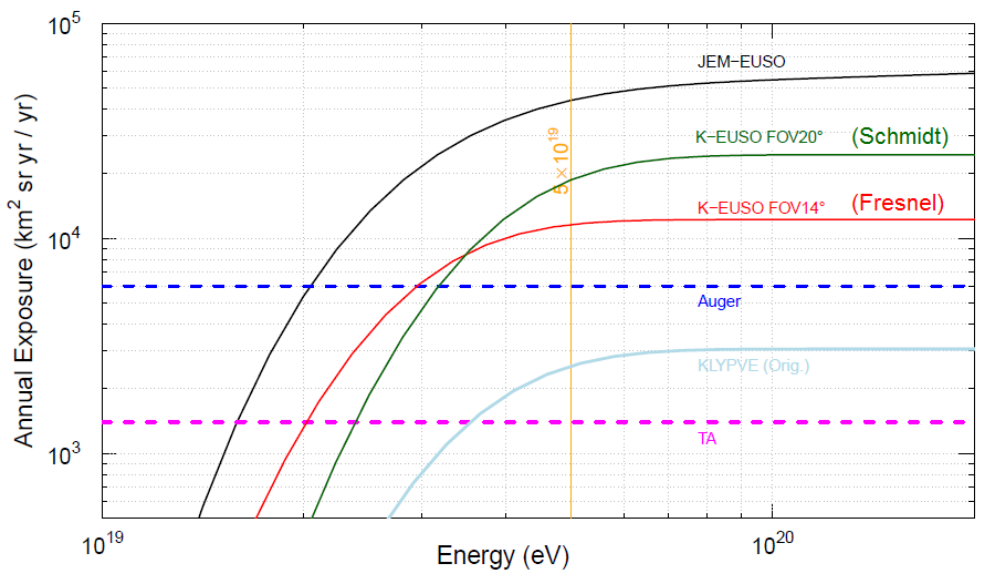

Fig. 7. Exposure comparison. 


\section{References}

[1]. M. Born E. Wolf, Principles of Optics, Pergamon Press, 6th Ed. Page 233-255.

[2]. W. Smith "Modern Lens Design", 2nd Ed. McGraw-Hill SPIE Press.

[3]. R. N. Wilson "Wide -field telescopes" in Reflecting Telescope Optics I, 2nd Ed., Astronomy and Astrophysics Library, Springer (2007).

[4]. E. M. Wormser "On the Design of Wide Angle Schmidt Optical Systems", JOSA, Vol. 40, No. 7 (1950).

[5]. Pierre Auger Collaboration, "The Fluorescence Detector of the Pierre Auger Observatory", arXiv:0907.4282v1 [astro-ph.IM] 24 Jul 2009.

[6]. R. Mirzoyan et al. "A $15^{\circ}$ wide field of view imaging air Cherenkov Telescope", Astroparticle Physics 31 (2009) 1-5.

[7]. P. Mazzinghi et al. "Large aperture and wide field of view space telescope for the detection of ultra high energy cosmic rays and neutrinos", Proc. "6th Internat. Conf. on Space Optics", ESTEC, Noordwijk, The Netherlands, 27-30 June 2006 (ESA SP-621, June 2006).

[8]. B. Schmidt, "Ein lichtstarkes komafreies Spiegelsystem," Central-Zeitung für Optik und Mechanik 52.2, pp. 25-26, (1931).

[9]. Zemax OpticStudio 16.5. OpticStudio is a registered Trademark of Zemax.

[10]. A. Romoli et al. "Schmidt camera correctors for large telescopes", Appl. Opt., Vol. 49, No. 15 (2010).

[11]. Y. Takizawa et al. "The TA-EUSO and EUSO-Balloon optics designs" 33rd ICRC (2013).

[12]. A. Zuccaro Marchi et al. "The JEM-EUSO optics design", 32nd ICRC, Beijing (2011).

[13]. E. H. Linfoot and E. Wolf "On the Corrector Plates of Schmidt Cameras", JOSA, Vol. 39, No. 9 (1949).

[14]. Dittman M.G., "Contamination scatter functions for stray-light analysis", Proc. of SPIE Vol. 4774, 99 (2002).

[15]. Harvey J.E. et al. "Total integrated scatter from surfaces with arbitrary roughness, correlation width and incident angles", Optical Engineering 51(1), 013402 (January 2012).

[16]. Irizar J. and Harnich B., "BRDF Prediction Using Surface Micro-roughness Measurements", ICSO (2012).

[17]. Stover J. C. "Optical scattering: measurement and analysis", Second edition SPIE Optical Engineering Press, (1995).

[18]. Wein S.J., "Small-angle scatter measurement," PhD Thesis, Univ. of Arizona, Tuscon (1989).

[19]. P. Mazzinghi et al. "An ultra-lightweight, Large aperture, deployable telescope for Advanced Lidar applications", A. Wilson, Ed., Proc. 6th Internat. Conf. on Space Optics, ESTEC, Noordwijk, The Netherlands, ISBN 93-9092-932-4, ISSN 1609-042X, ESA SP-621 (2006).

[20]. P. Mazzinghi et al. "Deployable, lightweight and large aperture space borne telescope for LIDAR based Earth observation", Proc. of SPIE Vol. 6750, 67500X, (2007).

[21]. F. Simonetti et al. "Large aperture telescope for advanced lidar system", Optical Engineering 49 (7), 073001 (2010).

[22]. http://www.schott.com/advanced_optics/english/products/optical-materials/zerodur-extremely-low-expansion-glass-ceramic/zerodur/index.html

[23]. R. Briguglio et al. "Laboratory demonstrator of a primary active mirror for space with the LATT: large aperture telescope technology”, Proc. SPIE 9904, Space Telescopes and Instrumentation (2016).

[24]. T. Strotman and Y. Tameda for the Telescope Array Collaboration: "Telescope Array Measurement of UHECR composition from stereoscopic fluorescence detection", Proc. 34th ICRC, The Hague, Netherlands (2015).

[25]. http://jem-euso.roma2.infn.it/?page_id=1058. 